Noname manuscript No.

(will be inserted by the editor)

\section{Damage evolution and debonding in hybrid laminates with a cohesive interfacial law}

\author{
Roberto Alessi . \\ Jacopo Ciambella . \\ Achille Paolone
}

the date of receipt and acceptance should be inserted later

\begin{abstract}
The hybridisation of fibres reinforced laminates, i.e., the combined use of two or more families of fibres, is an effective technique to achieve a pseudoductile response and overcome the inherent brittleness which limits the wider use of composite materials. In this paper, a one-dimensional analytical model for unidirectional hybrid laminates is derived. The model considers two elastic-brittle layers bonded together by a cohesive elasto-plastic-brittle interface. This formulation is applied to the study of the de-bonding and fracture of laminates under uniaxial loading and the results compared to experiments available from the open literature. This study shows that the proposed model provides a close fit to the experimental data and it is able to match accurately the crack patterns seen in the experiments. The model predicts four different failure mechanisms and is able to discriminate among them according to the geometrical and mechanical properties of the layers.
\end{abstract}

Keywords Hybrid composites · Fracture Mechanics · FRP · retrofitting

\section{R. Alessi}

Dipartimento di Matematica

Università di Roma La Sapienza

Piazzale Aldo Moro 5, 00185 Roma IT

R. Alessi · J. Ciambella . A. Paolone

Dipartimento di Ingegneria Strutturale e Geotecnica

Università di Roma La Sapienza

Via Eudossiana 18, 00184 Roma IT

J. Ciambella

Advanced Composites Centre for Innovation and Science (ACCIS)

University of Bristol

University Walk BS81TR Bristol UK

E-mail: jacopo.ciambella@uniroma1.it

\section{Introduction}

Although composite materials are increasingly used in all fields of engineering, their inherent brittleness is a significant issue that limits their wider use. Uni-Directional (UD) composite laminates are prone to a catastrophic failure when the ultimate tensile stress is reached. As a consequence, when used in applications for which the loading conditions are unpredictable, high safety coefficients are adopted with the drawback of drastically reducing their cost effectiveness. Thus, it is not surprising that most of the research in this field has been devoted to overcome this fundamental limitation and several strategies to improve the material ductility have been proposed including the use of ductile [6] or tapered fibers [25], woven or braided fabrics [11, 12, 20] and more generically the use of hybrid materials 22 , 21, 9, 24.

Among the others, the hybridisation of continuous fibers has been successfully applied to obtain a pseudoductile response in thin-ply laminates [8]. This technique is based on the combined use of two or more families of fibers, e.g., carbon/glass fibers, with different mechanical properties tuned to drive the failure of the interface within the plies and achieve the pseudoductile response much sought after.

All these techniques, however, ask for reliable models able to predict correctly the material behavior and guide the experimentalists towards the definition of new layups. Over the years, several idealised models were proposed in order to predict the strength of composite laminates [27, 18, 26, and have proved to be able to qualitatively match the experimental curves. In 10 . the strength of a structural member composed by two elastic layers bonded together by an adhesive layer is estimated by following an analytical approach. Similarly in [16, 17] strength and fracture patterns observed in a bilayer system subjected to thermal expansion are studied. Many works have investigated the strength of structural members reinforced with hybrid laminates through 1-D or 2-D numerical simulations [5, 23. More recently finite elements simulations were carried out in [13. to simulate the interface between two plies with different strengths by using cohesive elements; the authors introduced a Damage Mode Map (DMM), i.e. a 2D plot which describes the different failure scenarios in terms of the geometric properties of the layup. Four different failure mechanisms were accounted for all terminating with the failure of the high strength layer, namely: (i) diffuse delamination, (ii) multiple fractures of the low strain material, (iii) fragmentation in the low strain material followed by diffuse delamination, (iv) premature failure of the high strain material. All 
these failure modes are indeed experimentally observed which proves the map to be an effective design tool to aid the development of new composite layups. Based on previous results, the same authors proposed in [14] an analytical model: the main underlying hypothesis was assuming the interface to have a perfectly plastic response. From one hand, this choice allowed the derivation of a closed form expression of the ultimate tensile stresses in terms of the laminate parameters; from the other hand, it underestimates the typical crack length and requires the use of complex stochastic tools to predict the crack evolution in the low-strain layer.

In this work, we aim at providing a more accurate description of the laminate failure mechanisms by enforcing a simple yet effective cohesive law of the interface. Such a choice is representative of the real material and in fact leads to a more accurate prediction of the stress evolution within the laminate as well as of the crack spacing length observed in the experiments [7. Moreover, the cohesive law removes the indeterminacy of the crack locations in the low strain layer, which is usually overcome in rigid-perfectly plastic models by enforcing a stochastic distribution of the material parameters. A closed-form expression of the damage mode map is also derived to describe the different failure mechanism seen in the experimental data.

The plan of the paper is as follows. In Sec. 2 the overall laminate problem is introduced and it is shown that it can be reduced to the study of a unit-cell representative element. In Sec. 3 the response of the unit-cell subjected to an imposed external displacement is analyzed and the results are used in Sec. 4 to obtain the response of the entire laminate and highlight the different failure mechanisms that could occur. A comparison between the proposed model and the experimental results available from the open literature is carried out in Sec. 5. Finally conclusions are drawn in Sec. 6

\section{Position of the problem}

The UD hybrid laminate considered in this study is made of two homogeneous elastic-brittle layers with different failure strains and periodically alternating through the thickness Fig. 11a.

Due to the periodicity of the structure, the mechanical response of the laminate can be analysed by studying in a one-dimensional setting the response of the semi-periodic slice in Fig. 1p with length $L$ and thickness $h \ll L$; this slice consists of two layers with heights $h_{1}$ and $h_{2}$ respectively, bonded together by a cohesive interface whose thickness is assumed negligible. The first layer $\Omega_{1}$, denominated high-strain layer, has a higher failure strain compared to the second layer $\Omega_{2}$, therefore denominated low-strain layer. The hybrid laminate, and hence the semi-periodic slice, is subjected to a prescribed displacement $U_{\mathrm{L}}(t)$ applied to the rightend side whereas the left-end side is fixed. The external applied displacement is assumed to be monotonically increasing at a sufficiently low rate; as such, ratedependent and inertial effects can be neglected from the analysis.

For such a model, during the initial stage of the loading process, all layers experience the same homogeneous strain until the low-strain layer, assumed in the figure to be underneath, reaches its elastic limit and a first fracture appears. Being the two layers homogeneous, the position of this first crack is indeterminate by the model. The mechanical response of the fractured laminate changes abruptly and by further increasing the displacement, the different fracture scenarios (i)-(iv) described in the Introduction could occur. However, disregarding the exact failure mechanisms, the laminate is the superposition of several unit-cells (UCs) of generic length $\ell$ as shown in Fig. 11.

Each unit-cell (UC) represents a portion of the laminate with one end identified by the position at which the layers experience the same displacement and the other end corresponding to the neighbour position at which a fracture has occurred. The determination of the mechanical response of the UC in terms of the displacement prescribed to the high-strain layer allows the response of the entire laminate to be obtained by simple compatibility arguments as shown in the next sections.

\section{Unit-Cell response}

In this section, the mechanical response of the UC in Fig. 1. under a prescribed displacement at $\Omega_{1}$ is determined by assuming that both layers have an infinite strength, i.e., no further cracks occur apart from the one already in $\Omega_{2}$. The high- and the low-strain layers are assumed to have an elastic-brittle response with tensile moduli $\mathrm{E}_{1}, \mathrm{E}_{2}$ and ultimate strains $\bar{\varepsilon}_{2}<\bar{\varepsilon}_{1}$, respectively (Fig. 2a). Correspondingly, the ultimate stresses are $\bar{\sigma}_{1}:=\mathrm{E}_{1} \bar{\varepsilon}_{1}$ and $\bar{\sigma}_{2}:=\mathrm{E}_{2} \bar{\varepsilon}_{2}$.

The cohesive interface $\Gamma$, whose thickness is very thin compared to the other layers, is assumed to have an elasto-plastic-brittle response for which $\tau=\mu \delta$ if $\delta \leq \delta_{y}, \tau=\bar{\tau}$ if $\delta_{y}<\delta \leq \bar{\delta}$ and $\tau=0$ when $\delta>\bar{\delta}$ (Fig. 2p). Here $\delta(x):=u_{1}(x)-u_{2}(x)$ is the relative displacement between the two layers at a given position $x$, $\mu$ is the shear stiffness modulus (the adhesive shear modulus divided by the interface thickness which may embed also the contribution of the shear deformation of the cracked layer in the same spirit suggested in [19]), $\bar{\tau}$ 


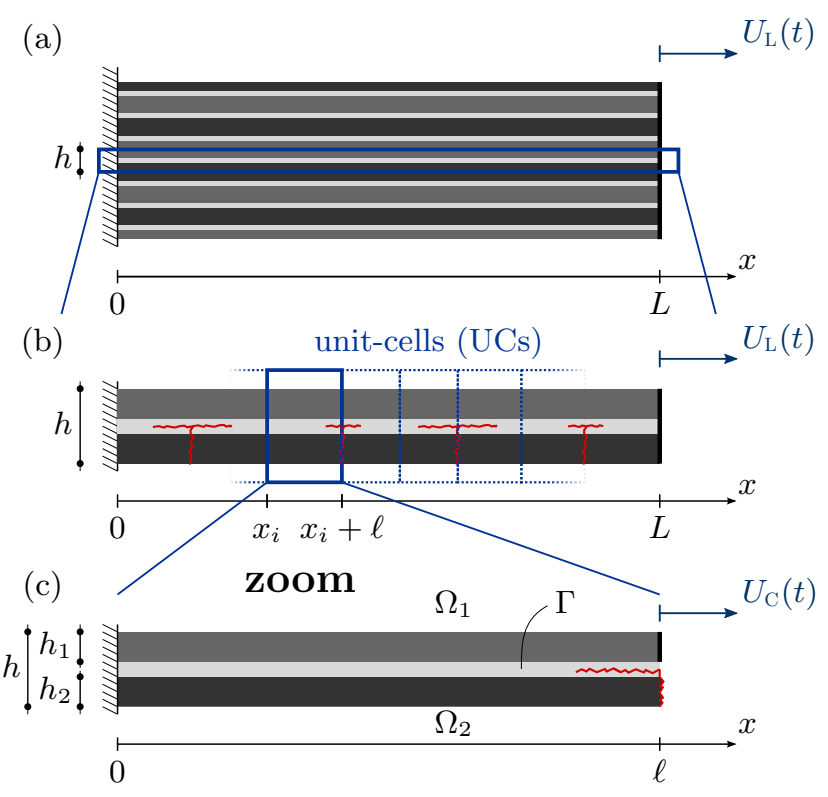

Fig. 1: Schematic representation of: (a) the hybrid laminate, (b) the one-dimensional model where fractures at the low strength layers and delamination at the interface have occurred, (c) the unit-cell model
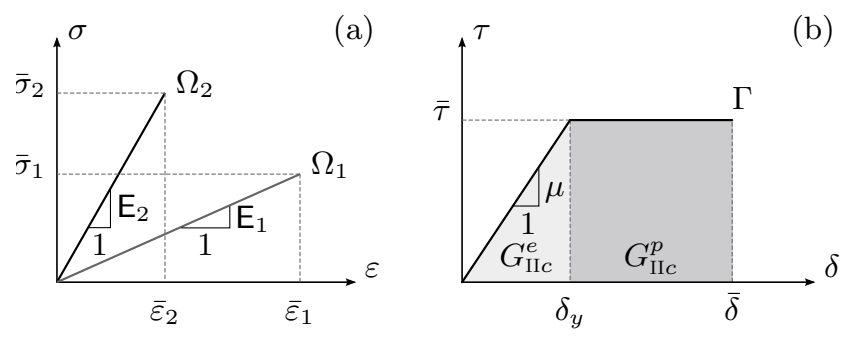

Fig. 2: Material response for: (a) the elastic layers, (b) the cohesive interface

and $\delta_{y}$ the shear yield stress and displacement respectively and $\bar{\delta}$ the debonding displacement. The interface mode II fracture toughness is the area under the curve $\tau(\bar{\delta})$, i.e., $G_{\mathrm{II} c}=\bar{\tau}\left(\bar{\delta}-\delta_{y} / 2\right)$. When the relative displacement $\delta$ between the layers is zero, the effects of the interface on the UC response are neglected since the adhesive thickness is evanescent and the tensile modulus of $\Gamma$ is assumed much lower than the one of the layers. Note that the model [15] is recovered when $\delta_{y}=0$.

The response of the $\mathrm{UC}$, whose length has been normalized to 1 , is characterized by the three different stages elastic, elasto-plastic and elasto-plastic-delaminated for an increasing prescribed dimensionless displacement $U=U_{\mathrm{C}}(t) / \ell$. Initially, the $\mathrm{UC}$ is in its elastic stage, Fig. 3 a, until the stress at the interface reaches its yield stress, namely $\tau=\bar{\tau}$. By continuing increasing the displacement, a plastic region is created which evolves to- (a) Elastic stage

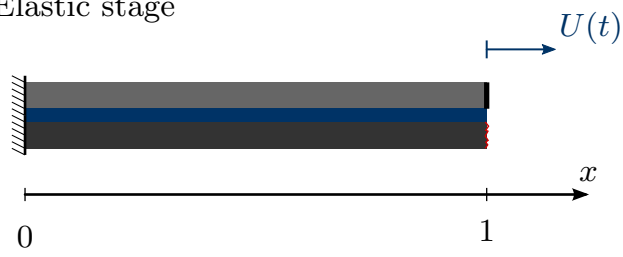

(b) Elastic-plastic stage

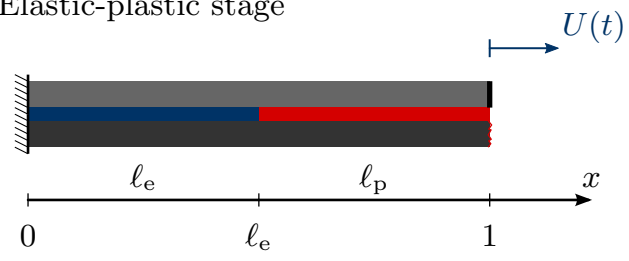

(c) Elastic-plastic-delaminated stage

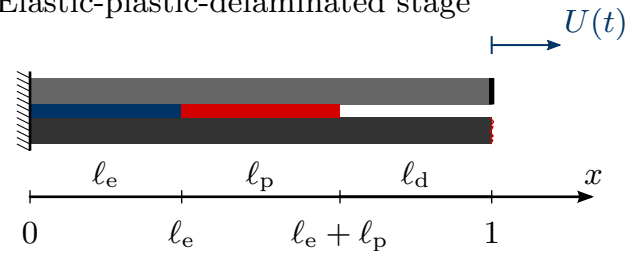

Fig. 3: Different stages of the UC loading process

wards the fixed end of the UC. This stage is therefore called elasto-plastic stage, Fig. 3 b, and lasts until the relative displacement of the two layers reaches the debonding limit, namely $\delta=\bar{\delta}$. Further increments of $U$ lead to the propagation of a delamination from the right-end side of the UC towards the fixed end which accordingly shifts and modifies the width of the plastic region. This latter stage is called elasto-plasticdelaminated stage, Fig. 3 .

In the next subsections each stage of the loading process is analyzed in detail, where all equations are presented in a dimensionless form through the following non-dimensional quantities:

$\hat{x}=x / \ell, \quad \hat{u}_{i}=u_{i} / \ell, \quad \hat{\delta}=\delta / \ell$

$\hat{\sigma}_{i}=\sigma_{i} / \mathrm{E}_{1}, \quad \hat{\tau}=\tau / \mathrm{E}_{1}$

This non-dimensional formulation will be used throughout the paper unless differently stated. For the sake of conciseness, the superimposed hat $\left(^{\wedge}\right)$ will be omitted in the following.

\subsection{Elastic stage}

During the initial stage of the loading process, both the layers experience the same deformation and hence the adhesive doesn't contribute to the composite response and stays unstressed. The apparent tensile modulus of the UC can be expressed in terms of the tensile moduli 
and the thicknesses of the layers as

$\mathrm{E}_{c}=\frac{\mathrm{E}_{1} h_{1}+\mathrm{E}_{2} h_{2}}{h}$

with $\mathrm{E}_{1}, \mathrm{E}_{2}$ being the elastic moduli in their dimensional forms.

This elastic stage ends up when the first crack appears, that is when the stress in the second layer reaches the failure stress $\bar{\sigma}_{2}$ or equivalently the corresponding overall strain $\bar{\varepsilon}_{2}$. In such a case, the configuration displayed in Fig 3 a can be studied. All the materials are still in their elastic regime but the interface starts playing a role in the UC response. The non-dimensional governing equations of the problem are:

$\left\{\begin{array}{ll}u_{1}^{\prime \prime}-\mathrm{F}\left(u_{1}-u_{2}\right)=0, & \text { in } \Omega_{1} \\ \mathrm{~K} u_{2}^{\prime \prime}+\mathrm{F}\left(u_{1}-u_{2}\right)=0, & \text { in } \Omega_{2}\end{array} \quad, x \in[0,1]\right.$

where the two non-dimensional constants $\mathrm{K}$ and $\mathrm{F}$ are given by

$\mathrm{K}:=\mathrm{E}_{2} h_{2} /\left(\mathrm{E}_{1} h_{1}\right), \quad \mathrm{F}:=\mu \ell^{2} /\left(\mathrm{E}_{1} h_{1}\right)$

representing, respectively, the relative stiffness of the layers and a generalized elastic interface shear stiffness. The boundary conditions are given by $u_{1}(0)=0$, $u_{2}(0)=0, u_{1}(1)=U$ and $u_{2}^{\prime}(1)=0$. By introducing the dimensionless parameters $\alpha=\mathrm{F}(\mathrm{K}+1) / \mathrm{K}$ and $\beta=\mathrm{F} / \mathrm{K}$, the solution of (3) in terms of the dimensionless stresses is

$\sigma_{1}(x)=\frac{\beta \sqrt{\alpha} \cosh (\sqrt{\alpha})+(\alpha-\beta) \cosh (x \sqrt{\alpha})}{\beta \sqrt{\alpha} \cosh (\sqrt{\alpha})+(\alpha-\beta) \sinh (\sqrt{\alpha})} U$,

$\sigma_{2}(x)=\frac{\sqrt{\alpha}(\alpha-\beta)(\cosh (\sqrt{\alpha})-\cosh (x \sqrt{\alpha}))}{\beta \sqrt{\alpha} \cosh (\sqrt{\alpha})+(\alpha-\beta) \sinh (\sqrt{\alpha})} U$

whereas the dimensionless shear stress at the interface is

$\tau(x)=\frac{\sqrt{\alpha}(\alpha-\beta) \sinh (x \sqrt{\alpha})}{\beta \sqrt{\alpha} \cosh (\sqrt{\alpha})+(\alpha-\beta) \sinh (\sqrt{\alpha})} U$

Due to the occurrence of the crack, the stress in the second layer is released and becomes zero at the crack tip, i.e., at $x=1$. As such, the stress at the interface is maximum in correspondence of the crack, whereas decreases monotonically towards $x=0$.

An example of the stress evolution along the UC length is shown in Fig. 4 for $\mathrm{K}=1, \mathrm{~F}=3$ and $U=$ 1. The figure shows that $\sigma_{1}$ and $\tau$ are maximum at the crack location whereas $\sigma_{2}$ is maximum at $x=0$; the interface yield limit is therefore reached at $x=1$ and the corresponding limit displacement $U_{y}$ can be evaluated from (5).

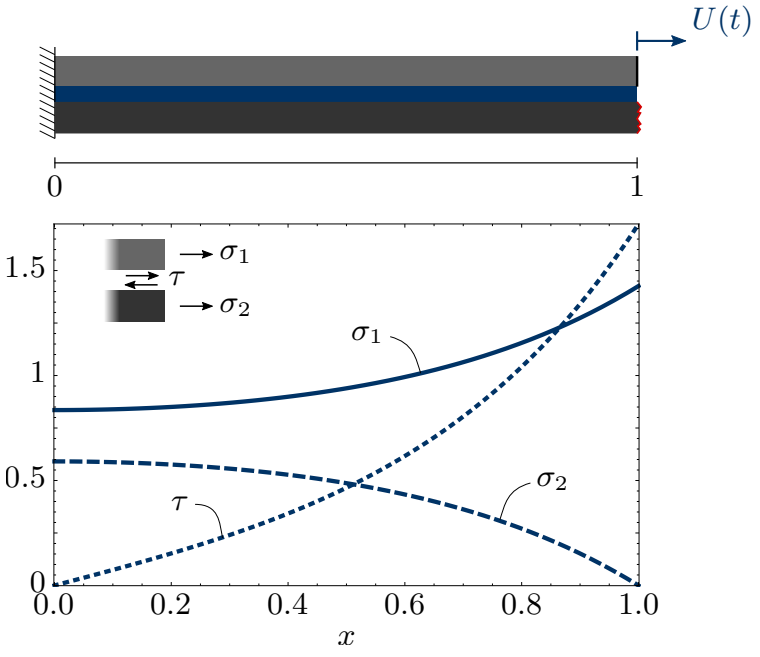

Fig. 4: Stresses distribution along the UC during the elastic stage for $\mathrm{K}=1, \mathrm{~F}=3$ and $U=1$

The apparent dimensionless tensile modulus of the $\mathrm{UC}$, defined as $\mathrm{E}_{c}:=\sigma_{1}(1) / U$, is equal to

$\mathrm{E}_{c}=\frac{\alpha \sqrt{\alpha}}{\sqrt{\alpha} \beta+(\alpha-\beta) \tanh (\sqrt{\alpha})}$

The elastic stage ends up when the interface relative displacement $\delta$ reaches the yield limit $\delta_{y}$, which implies the stresses $\sigma_{1}(1)$ and $\sigma_{2}(0)$ to be

$\bar{\sigma}_{1}^{\mathrm{ep}}=\delta_{y} \sqrt{\alpha} \operatorname{coth}(\sqrt{\alpha})$

$\bar{\sigma}_{2}^{\mathrm{ep}}=\frac{\beta \delta_{y} \tanh (\sqrt{\alpha} / 2)}{\sqrt{\alpha}}$

\subsection{Elasto-plastic stage}

Once the interface has started yielding, the elasto-plastic front evolves from the right-end side towards the leftend side of the UC till the debonding strain $\bar{\delta}$ is reached. This configuration is schematically depicted in Fig. $3 \mathrm{p}$ where the elastic and plastic regions, respectively of dimensionless lengths $\ell_{\mathrm{e}}$ and $\ell_{\mathrm{p}}$ with $\ell_{\mathrm{e}}+\ell_{\mathrm{p}}=1$, are highlighted.

In this case, the problem can be split into two subdomains, with the corresponding equilibrium equations given by

$\left\{\begin{array}{ll}u_{1 \mathrm{e}}^{\prime \prime}-\mathrm{F}\left(u_{1 \mathrm{e}}-u_{2 \mathrm{e}}\right)=0 & \text { in } \Omega_{1} \\ \mathrm{~K} u_{2 \mathrm{e}}^{\prime \prime}+\mathrm{F}\left(u_{1 \mathrm{e}}-u_{2 \mathrm{e}}\right)=0 & \text { in } \Omega_{2}\end{array} \quad, x \in\left[0, \ell_{e}\right]\right.$

$\left\{\begin{array}{ll}u_{1 \mathrm{p}}^{\prime \prime}-\bar{\tau}=0 & \text { in } \Omega_{1} \\ \mathrm{~K} u_{2 \mathrm{p}}^{\prime \prime}+\bar{\tau}=0 & \text { in } \Omega_{2}\end{array} \quad, x \in\left[\ell_{e}, 1\right]\right.$ 
and the corresponding boundary conditions at $x=0$ (the fixed boundary):

$u_{1 \mathrm{e}}(0)=0, \quad u_{2 \mathrm{e}}(0)=0$

at $x=\ell_{\mathrm{e}}$ (continuity):

$u_{1 \mathrm{e}}\left(\ell_{\mathrm{e}}\right)=u_{1 \mathrm{p}}\left(\ell_{\mathrm{e}}\right), \quad u_{2 \mathrm{e}}\left(\ell_{\mathrm{e}}\right)=u_{2 \mathrm{p}}\left(\ell_{\mathrm{e}}\right)$,

$u_{1 \mathrm{e}}^{\prime}\left(\ell_{\mathrm{e}}\right)=u_{1 \mathrm{p}}^{\prime}\left(\ell_{\mathrm{e}}\right), \quad u_{2 \mathrm{e}}^{\prime}\left(\ell_{\mathrm{e}}\right)=u_{2 \mathrm{p}}^{\prime}\left(\ell_{\mathrm{e}}\right)$;

and at $x=1$, where the applied displacement is prescribed:

$u_{1 \mathrm{p}}(1)=U, \quad u_{2 \mathrm{p}}^{\prime}(1)=0$

The lengths of the two regions $\ell_{\mathrm{e}}$ and $\ell_{\mathrm{p}}$ are determined by assuming that the interface at the elastoplastic transition point attains the yield condition, i.e.,

$\left(u_{1 \mathrm{e}}\left(\ell_{\mathrm{e}}\right)-u_{2 \mathrm{e}}\left(\ell_{\mathrm{e}}\right)\right) \mu=\bar{\tau}$

which gives an implicit expression of $\ell_{\mathrm{e}}$ in terms of the applied displacement $U_{\mathrm{ep}}\left(\ell_{\mathrm{e}}\right)$ (Eq. (27) in Appendix). This function allows the governing equations (11) to be solved by parameterizing the problem with $\ell_{\mathrm{e}}$ and deducing a posteriori the corresponding applied displacement $U=U_{\mathrm{ep}}\left(\ell_{\mathrm{e}}\right)$. The solution in the elastic region is the same as the one in Sec. 3.1 but properly rescaled whereas the stresses in the plastic region are linear and the shear stress constant at the interface.

An example of the stress profiles along the UC is given in Fig 5. As for the elastic stage, the stresses in $\Omega_{1}$ and $\Omega_{2}$ are maxima at $x=1$ and $x=0$, respectively. The elasto-plastic stage lasts until the shear strain reaches the ultimate limit at $x=1$, that is when $u_{1 \mathrm{p}}(1)-u_{2 \mathrm{p}}(1)=\bar{\delta}$ (Eq. 28 in Appendix). The corresponding limit length $\bar{\ell}_{\mathrm{e}}$ and limit displacement $\bar{U}$ are evaluated by using (27) with the expression (28). Correspondingly, the maximum stress in $\Omega_{1}$ is $\sigma_{1}(1)=\bar{\sigma}_{1}^{\text {pd }}$ whereas the maximum stress in $\Omega_{2}$ is $\sigma_{2}(0)=\bar{\sigma}_{2}^{\mathrm{pd}}$. The quantities $\bar{\sigma}_{1}^{\text {pd }}$ and $\bar{\sigma}_{2}^{\text {pd }}$ are respectively given in Appendix by the equations (29) and (30).

\subsection{Elasto-plastic-delaminated stage}

The final stage of the loading process, depicted in Fig. 3. is characterized by three regions: elastic, plastic and delaminated. Indeed, as soon as the interface relative displacement $\delta$, which is maximum at $x=1$, attains the limit value $\bar{\delta}$, debonding is triggered and the delaminated region starts extending towards the left-end side of the UC with length $\ell_{\mathrm{d}}$. Consequently, the elastoplastic region has length $\ell_{\mathrm{e}}+\ell_{\mathrm{p}}=1-\ell_{\mathrm{d}}$, Fig. 3. In the delaminated region, the stress is not transferred between the layers, thus the structural problem can be

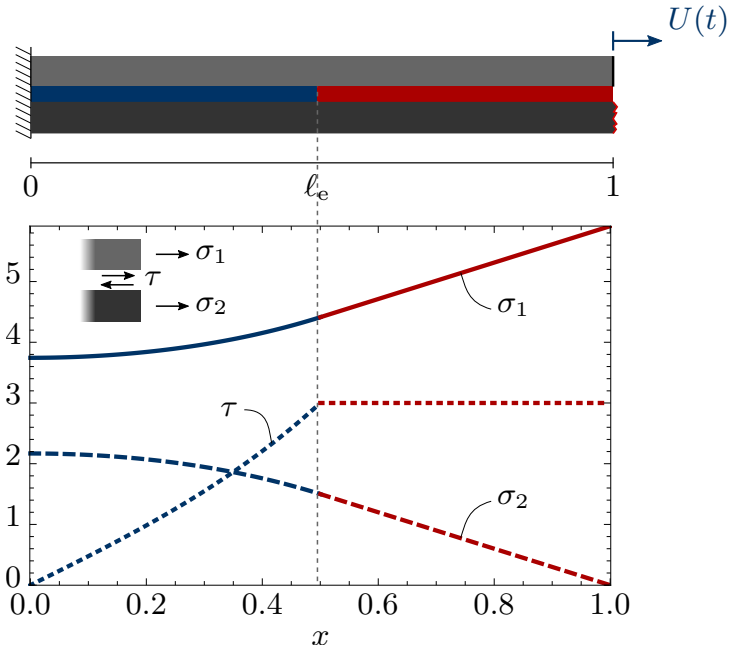

Fig. 5: Stresses distribution along the UC length during the elasto-plastic stage for $\mathrm{K}=1, \mathrm{~F}=3$ and $U=$ $U_{\text {ep }}\left(\ell_{\mathrm{e}}=0.5\right)$

decomposed into two subproblems. The former, associated to the subdomain $x \in\left[0, \ell_{\mathrm{e}}+\ell_{\mathrm{p}}\right]$, can be studied by rescaling the elasto-plastic problem already investigated in Sec. 3.2. The latter, which is associated to the subdomain $x \in\left[\ell_{\mathrm{e}}+\ell_{\mathrm{p}}, 1\right]$, is a linear elastic problem in which all loading is carried by $\Omega_{1}$ whereas $\Omega_{2}$ is unstressed and, therefore, rigidly shifted (see Fig. 6).

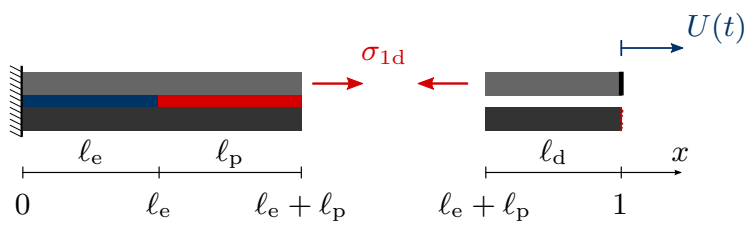

Fig. 6: Problem decomposition in the elasto-plasticdelaminated stage

The equilibrium equations for this stage are

$$
\begin{aligned}
& \left\{\begin{array}{ll}
u_{1 \mathrm{e}}^{\prime \prime}-\mathrm{F}\left(u_{1 \mathrm{e}}-u_{2 \mathrm{e}}\right)=0 & \text { in } \Omega_{1} \\
\mathrm{~K} u_{2 \mathrm{e}}^{\prime \prime}+\mathrm{F}\left(u_{1 \mathrm{e}}-u_{2 \mathrm{e}}\right)=0 & \text { in } \Omega_{2}
\end{array}, x \in\left[0, \ell_{\mathrm{e}}\right]\right. \\
& \left\{\begin{array}{ll}
u_{1 \mathrm{p}}^{\prime \prime}-\bar{\tau}=0 & \text { in } \Omega_{1} \\
\mathrm{~K} u_{2 \mathrm{p}}^{\prime \prime}+\bar{\tau}=0 & \text { in } \Omega_{2}
\end{array}, x \in\left[\ell_{\mathrm{e}}, \ell_{\mathrm{p}}\right]\right.
\end{aligned}
$$

for the first subproblem, whereas for the second subproblem

$\left\{\begin{array}{ll}u_{1 \mathrm{~d}}^{\prime \prime}=0 & \text { in } \Omega_{1} \\ \mathrm{~K} u_{2 \mathrm{~d}}^{\prime \prime}=0 & \text { in } \Omega_{2}\end{array} \quad, x \in\left[\ell_{\mathrm{e}}+\ell_{\mathrm{p}}, 1\right]\right.$ 


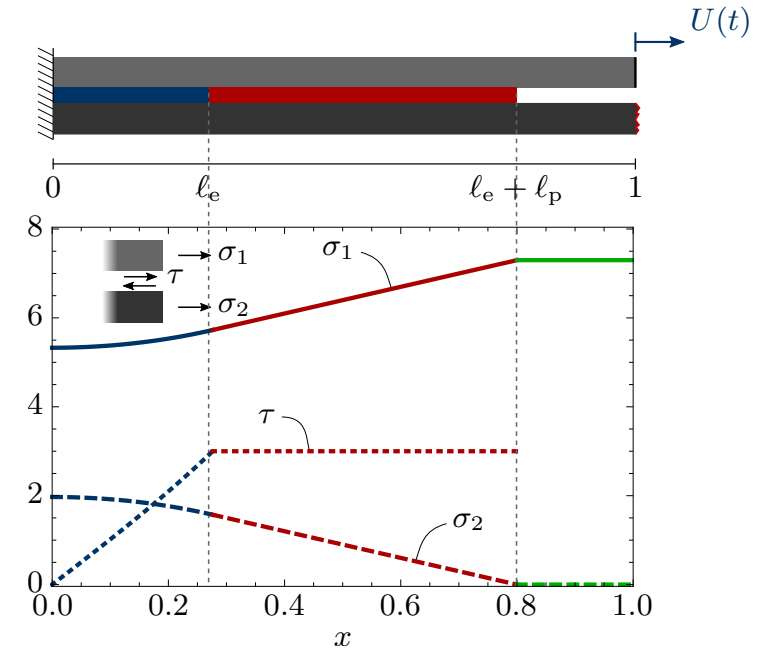

Fig. 7: Stresses distribution along theUC length during the elasto-plastic-delaminated stage for $\mathrm{K}=1, \mathrm{~F}=3$ and $U=U\left(\ell_{\mathrm{d}}=0.2\right)$

with boundary conditions at $x=0$ (the fixed boundary):

$u_{1 \mathrm{e}}(0)=0, \quad u_{2 \mathrm{e}}(0)=0$

at $x=\ell_{\mathrm{e}}$ (continuity):

$u_{1 \mathrm{e}}\left(\ell_{\mathrm{e}}\right)=u_{1 \mathrm{p}}\left(\ell_{\mathrm{e}}\right), \quad u_{2 \mathrm{e}}\left(\ell_{\mathrm{e}}\right)=u_{2 \mathrm{p}}\left(\ell_{\mathrm{e}}\right)$,

$u_{1 \mathrm{e}}^{\prime}\left(\ell_{\mathrm{e}}\right)=u_{1 \mathrm{p}}^{\prime}\left(\ell_{\mathrm{e}}\right), \quad u_{2 \mathrm{e}}^{\prime}\left(\ell_{\mathrm{e}}\right)=u_{2 \mathrm{p}}^{\prime}\left(\ell_{\mathrm{e}}\right) ;$

at $x=\ell_{\mathrm{e}}+\ell_{\mathrm{p}}$ (continuity):

$u_{1 \mathrm{p}}\left(\ell_{\mathrm{e}}+\ell_{\mathrm{p}}\right)=u_{1 d}\left(\ell_{\mathrm{e}}+\ell_{\mathrm{p}}\right)$,

$u_{2 \mathrm{p}}\left(\ell_{\mathrm{e}}+\ell_{\mathrm{p}}\right)=u_{2 d}\left(\ell_{\mathrm{e}}+\ell_{\mathrm{p}}\right)$,

$u_{1 \mathrm{p}}^{\prime}\left(\ell_{\mathrm{e}}+\ell_{\mathrm{p}}\right)=u_{1 d}^{\prime}\left(\ell_{\mathrm{e}}+\ell_{\mathrm{p}}\right)$,

$u_{2 \mathrm{p}}^{\prime}\left(\ell_{\mathrm{e}}+\ell_{\mathrm{p}}\right)=u_{2 d}^{\prime}\left(\ell_{\mathrm{e}}+\ell_{\mathrm{p}}\right)$

and at $x=1$, where the applied displacement is prescribed:

$u_{1 \mathrm{~d}}(1)=U, \quad u_{2 \mathrm{~d}}^{\prime}(1)=0$

By using Eq. (16), the solution of the second subproblem is

$\sigma_{1 \mathrm{~d}}=$ const. $\quad$ and $\quad \sigma_{2 \mathrm{~d}}=0$

which coupled with the solution of the first problem 15a-15b), derived in the previous section, allows the stress evolution in the entire UC parametrized with respect to $\ell_{\mathrm{d}}$ to be obtained. An example of the stress profiles in this stage is given in Fig. 7

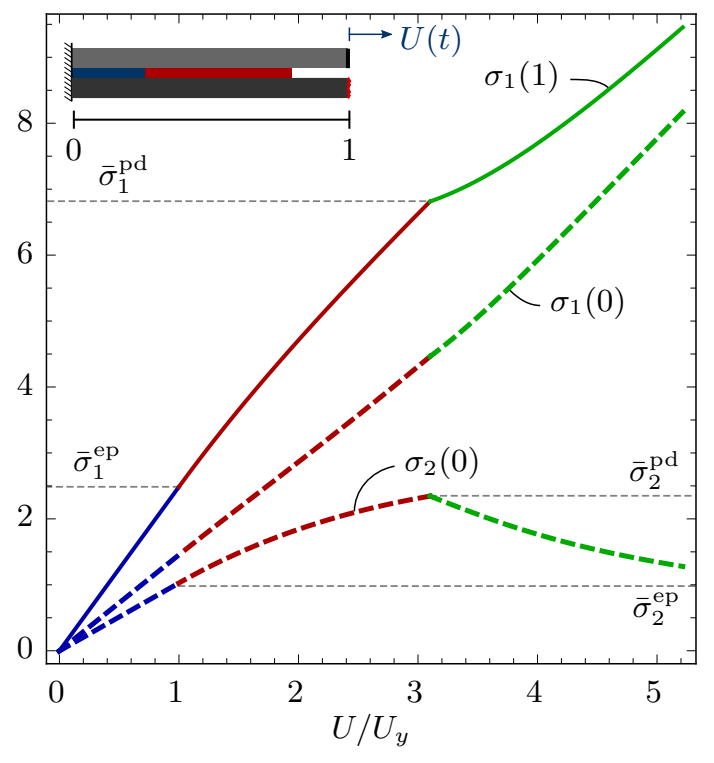

Fig. 8: Example of stress evolution during the elastoplastic-debonding stage for $\mathrm{K}=1, \mathrm{~F}=3, \delta_{y}=1, \bar{\delta}=$ 3 and $\ell_{\mathrm{d}}=0.2$. The colors refer to the stages, blue for elastic, red for elasto-plastic and green for elastoplastic-delaminated

\subsection{Global response of the UC}

Previous solutions are used here to derive the global response of the UC once the assumption of having infinitely resistant layers is removed.

The maximum stresses in $\Omega_{1}$ and $\Omega_{2}$ are attained for each loading stage at $x=0$ and $x \in\left[\ell_{\mathrm{e}}+\ell_{\mathrm{p}}, 1\right]$, respectively; their values can be calculated from Eqs. (9)-(10) and Eqs. (29)-(30) which by comparison with the corresponding limit stresses $\bar{\sigma}_{1}$ and $\bar{\sigma}_{2}$ determine the state of the UC, e.g., elastic, elasto-plastic or elasto-plasticdelaminated. It is worth remarking that the stress $\sigma_{1}(1)$ monotonically increases with the displacement whereas $\sigma_{2}(0)$ increases during the elasto-plastic stage but decreases during the propagation of the delamination, meaning that the capability of the interface to transfer stresses between the layers is progressively reduced. As a consequence, the failure of the low-strain layer $\Omega_{2}$ (fragmentation) can only occur during the elastic- or elastoplastic stages. The evolution of the stress within the layers for an increasing displacement is given in Fig. 8 for $\mathrm{K}=1, \mathrm{~F}=3, \delta_{y}=1, \bar{\delta}=3$ and $\ell_{\mathrm{d}}=0.2$.

According to whether the low- or high-strain layer reaches the ultimate strength first, several evolution scenarios in the response of the UC may occur. If during the elasto-plastic stage the low-strain material $\Omega_{2}$ reaches the ultimate strength, fragmentation occurs which could possibly lead to a diffuse delamination. On the 
contrary, if the high-strain material $\Omega_{1}$ fails first, the entire UC collapses.

\section{Laminate response}

The construction of the laminate global response can be algorithmically derived from the UC response by taking into account all the possible failure scenarios.

Initially the undamaged laminate is elastically loaded until the uniform strain in $\Omega_{2}$ reaches the critical value $\bar{\varepsilon}_{2}$ and a crack appears. The corresponding stress and strain, normally indicated as yield stress and yield strain, are

$\varepsilon_{\mathrm{Y}}=\bar{\varepsilon}_{2}, \quad$ and $\quad \sigma_{\mathrm{Y}}=\mathrm{E}_{c} \bar{\varepsilon}_{2}$

with $\mathrm{E}_{c}$ being the apparent tensile modulus of the undamaged laminate in (2).

Having assumed the two layers homogeneous with no imperfections, the position of the first crack is indeterminate. However, the presence of the crack allows the fractured laminate to be regarded as two UCs in series with possibly different lengths, whose response is completely known by the analysis carried out in the previous section. For an increasing applied displacement, the laminate response is given by the response of the two UCs until the ultimate strength limit is reached either in the high-strain or in the low-strain layer. In the former case, the entire laminate fails. In the latter case instead, another fracture in $\Omega_{2}$ occurs where $\sigma_{2}$ is maximum, i.e., at the fixed end of the longest UC. Correspondingly, the laminate can be analyzed as a three UCs chain and the previous analysis can be repeated leading to an increasing number of UCs until the highstrain layer reaches its ultimate strength.

It is worth noting that for a $n$-UCs chain the maximum stress in the low-strain layer is attained either at the center of the furthest contiguous cracks or at one laminate end. This last situation is likely to occur if the distance between the laminate end and the closest crack is larger than half of the size of the largest distance between two contiguous cracks. Moreover, no further fracture of $\Omega_{2}$ could occur if the delamination of the interface has started. This property guarantees that diffuse delamination is most likely to be triggered.

One of the objective of the present analysis was the accurate prediction of the different failure modes of the laminate; this is indeed a key feature to assess its pseudo-ductile performance. In this respect the damage mode map (DMM) introduced in [13] is an effective design tool and allows the different failure scenarios to be outline in a $2 \mathrm{D}$ plot in terms of the geometric properties of the composite layup. In particular,

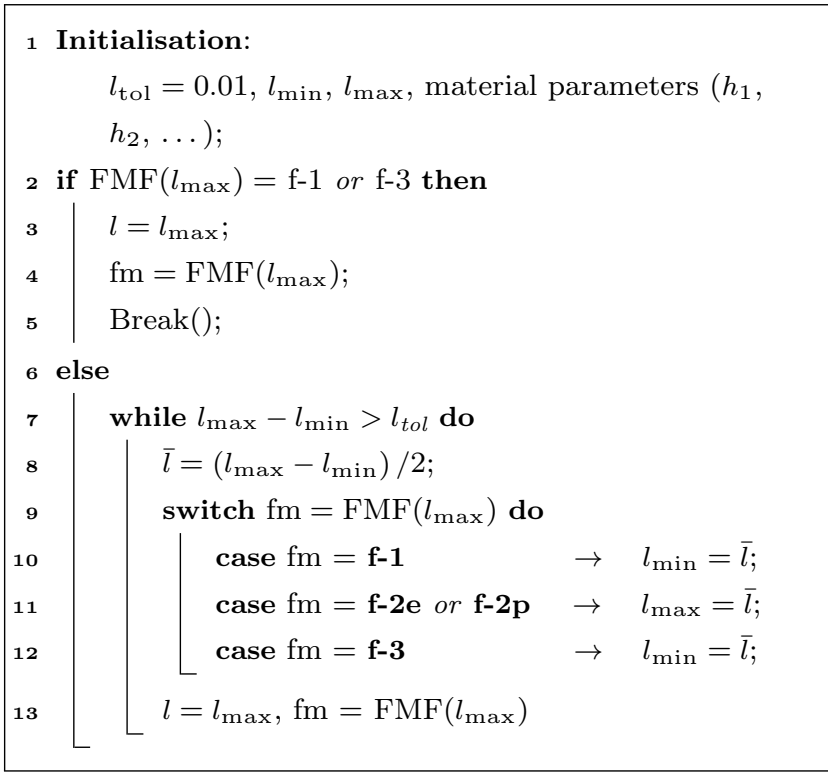

Fig. 9: The failure-mode prediction algorithm (FMPA). In the algorithm $\mathrm{fm}$ stands for failure mode and $\mathrm{f}-1 / 2 / 3$ represent the different failure mechanisms described in Tab. 1. The failure mechanism function (FMF) is defined in the Appendix, Fig. 13

the DMM is usually plotted against the relative thickness $h_{2} / h$ and the absolute thickness $h_{2}$. This choice is useful to give a very effective and clear representation of the four regions that identify the failure mechanisms: (C) Catastrophic delamination, (D) fragmentation and diffuse Delamination, (F) Fragmentation and (P) Premature failure of the high strain material. The curves that limit each region are shown in Fig. 10 and are labeled $\mathcal{C}^{\mathrm{CP}}, \mathcal{C}^{\mathrm{DF}}$ for the case of incipient debonding and $\mathcal{C}^{\mathrm{CD}}, \mathcal{C}^{\mathrm{FP}}$ for the case of of fragmentation.

The base idea for assembling the DMM is the failuremode prediction algorithm (FMPA) shown in Fig. 9 and based on a trial-error scheme. The algorithm seeks the maximum UC length at which the ultimate failure mode occurs, namely a complete delamination or the failure of the high-strain layer. More specifically, the function FMPA is used to determine the minimum length at which two different failure mechanisms simultaneously occur; the full DMM is recovered by spanning the entire range of admissible layer heights.

The closed form expressions for the limit curves cannot be obtained with an elasto-plastic interface. Nevertheless, it can be shown numerically that these curves coincide with those of an elastic-brittle interface model $\left(\delta_{y}=\bar{\delta}\right)$ with the same fracture toughness and maximal shear stress. In this latter case the solution can be explicitly found by deriving the expression of the max- 
imum elastic stress (force) in $\Omega_{1}$ at which each failure mechanism occurs; the limit curves can be determined by imposing that two failure mechanisms simultaneously occur.

\begin{tabular}{cl}
\hline $\mathbf{f - 1}$ & high-strain layer failure \\
\hline $\mathbf{f - 2 e}$ & $\begin{array}{l}\text { low-strain layer failure (fragmentation) } \\
\text { with elastic interface }\end{array}$ \\
\hline $\mathbf{f - 2} \mathbf{p}$ & $\begin{array}{l}\text { low-strain layer failure (fragmentation) } \\
\text { with plastic interface }\end{array}$ \\
\hline $\mathbf{f - 3}$ & interface delamination \\
\hline
\end{tabular}

Table 1: Different failure modes and their corresponding labels

The failure of the high strain layer occurs when the dimensionless force $s_{1}:=\sigma_{1} / E_{1}$ equals the limit value $\bar{s}_{1}:=\bar{\sigma}_{1} / \mathrm{E}_{1}$, namely

$s_{1}=\bar{s}_{1}$

Similarly, the failure of the low strain layer occurs when the dimensionless force $\bar{s}_{2}=\bar{\sigma}_{2} h_{2} /\left(\mathrm{E}_{1} h_{1}\right)$, which corresponds to a fragmentation of the composite, is attained. The explicit condition in terms of the non-dimensional material parameters and the force $s_{1}$ reads

$\alpha \bar{s}_{2}+(\alpha-\beta)(\operatorname{sech}(\sqrt{\alpha})-1) s_{1}=0$

A delamination of the interface occurs if $u_{1}-u_{2}=\bar{\delta}$, which leads to the condition, in terms of the dimensionless material parameters and the force $s_{1}$,

$s_{1}-\sqrt{\alpha} \operatorname{coth}(\sqrt{\alpha}) \bar{\delta}=0$

Based on previous results, the limit curves are hereafter expressed by making explicit $h_{2}$ as a function of $h_{1}$ at fixed material parameters:

curve $\mathcal{C}^{\mathrm{CP}}$ is obtained by assuming the contemporary occurrence of debonding and the high-strain layer failure, hence conditions $(\mathbf{f}-\mathbf{1}$ and $\mathbf{f - 3}$, for a laminate of infinite length. This leads to the explicit law

$$
h_{2}^{\mathrm{CP}}=-\frac{\mathrm{E}_{1}^{2} \mu h_{1} \bar{\delta}^{2}}{\mathrm{E}_{2}\left(\mathrm{E}_{1} \mu \bar{\delta}^{2}-h_{1} \bar{\sigma}_{1}^{2}\right)}
$$

curve $\mathcal{C}^{\mathrm{CD}}$ is obtained by assuming the contemporary occurrence of debonding and fragmentation, hence conditions $\mathbf{f - 2}$ and $\mathbf{f - 3}$, for a laminate of infinite length. This leads to the explicit law

$$
h_{2}^{\mathrm{CD}}=\frac{\bar{\sigma}_{2} \sqrt{\mathrm{E}_{1} h_{1}\left(4 \mathrm{E}_{2}^{2} \mu \bar{\delta}^{2}+\mathrm{E}_{1} h_{1} \bar{\sigma}_{2}^{2}\right)}-\mathrm{E}_{1} h_{1} \bar{\sigma}_{2}^{2}}{2 \mathrm{E}_{2} \bar{\sigma}_{2}^{2}}
$$

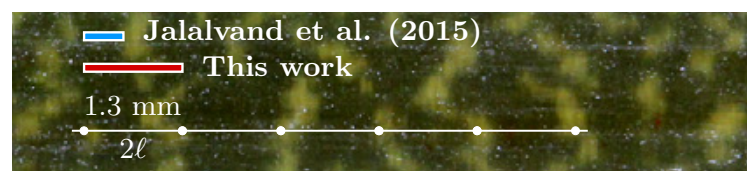

(a) Layup $1\left(\mathrm{G}_{2} \mathrm{CG}_{2}\right)$

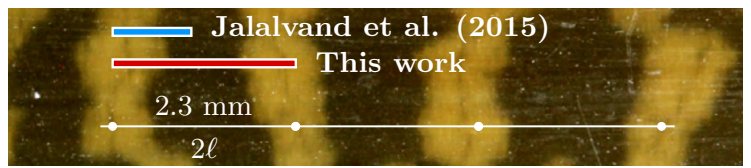

(b) Layup $2\left(\mathrm{G}_{2} \mathrm{C}_{2} \mathrm{G}_{2}\right)$

Fig. 11: Quasi-periodic crack patterns observed in the experiments in [8] for two different layups. Black and yellow regions represent the unbounded and bounded interfaces, respectively. Blue and red bars correspond to the predicted analytical lengths of Tab. 3

curve $\mathcal{C}^{\mathrm{DF}}$ is obtained by assuming the contemporary occurrence of debonding and the high-strain layer failure, hence conditions $(\mathbf{f - 1}$ and $(\mathbf{f - 3}$ but within a fragmentation regime $\mathbf{f - 2}$. This leads to the explicit law

$$
\begin{aligned}
& h_{2}^{\mathrm{DF}}=\frac{2 \mathrm{E}_{2} h_{1} \bar{\sigma}_{1} \bar{\sigma}_{2}-\mathrm{E}_{1} h_{1} \bar{\sigma}_{2}^{2}}{2 \mathrm{E}_{2} \bar{\sigma}_{2}^{2}}- \\
& \frac{\sqrt{\left(2 \mathrm{E}_{2} h_{1} \bar{\sigma}_{1} \bar{\sigma}_{2}-\mathrm{E}_{1} h_{1} \bar{\sigma}_{2}^{2}\right)^{2}-4 \mathrm{E}_{1} \mathrm{E}_{2}^{2} \mu h_{1} \bar{\delta}^{2} \bar{\sigma}_{2}^{2}}}{2 \mathrm{E}_{2} \bar{\sigma}_{2}^{2}}
\end{aligned}
$$

curve $\mathcal{C}^{\mathrm{FP}}$ is obtained by assuming the contemporary occurrence of fragmentation and high strain material failure, hence conditions $\mathbf{f - 1}$ and $\mathbf{f - 2}$, for a laminate of infinite length. This leads to the explicit law

$h_{2}^{\mathrm{FP}}=\frac{\mathrm{E}_{2} h_{1} \bar{\sigma}_{1}-\mathrm{E}_{1} h_{1} \bar{\sigma}_{2}}{\mathrm{E}_{2} \bar{\sigma}_{2}}$

An example of the DMM and respective limit curves is shown in Fig. 10 with the constitutive parameters of Tab. 2a and Tab. 2b.

\section{Results and Discussion}

In this section, the prediction of the model is compared to some experimental results in [8]. In particular, the analysis is focused on two layups, made of thin glass $\left(\Omega_{1}\right)$ and carbon $\left(\Omega_{2}\right)$ plies, for which pictures at failure of the upper surface are shown in Fig. 11. The failure mechanism and the presence of bounded and unbounded interfaces is clearly shown in the pictures as well as the characteristic crack spacing dependent upon the laminate layup. 


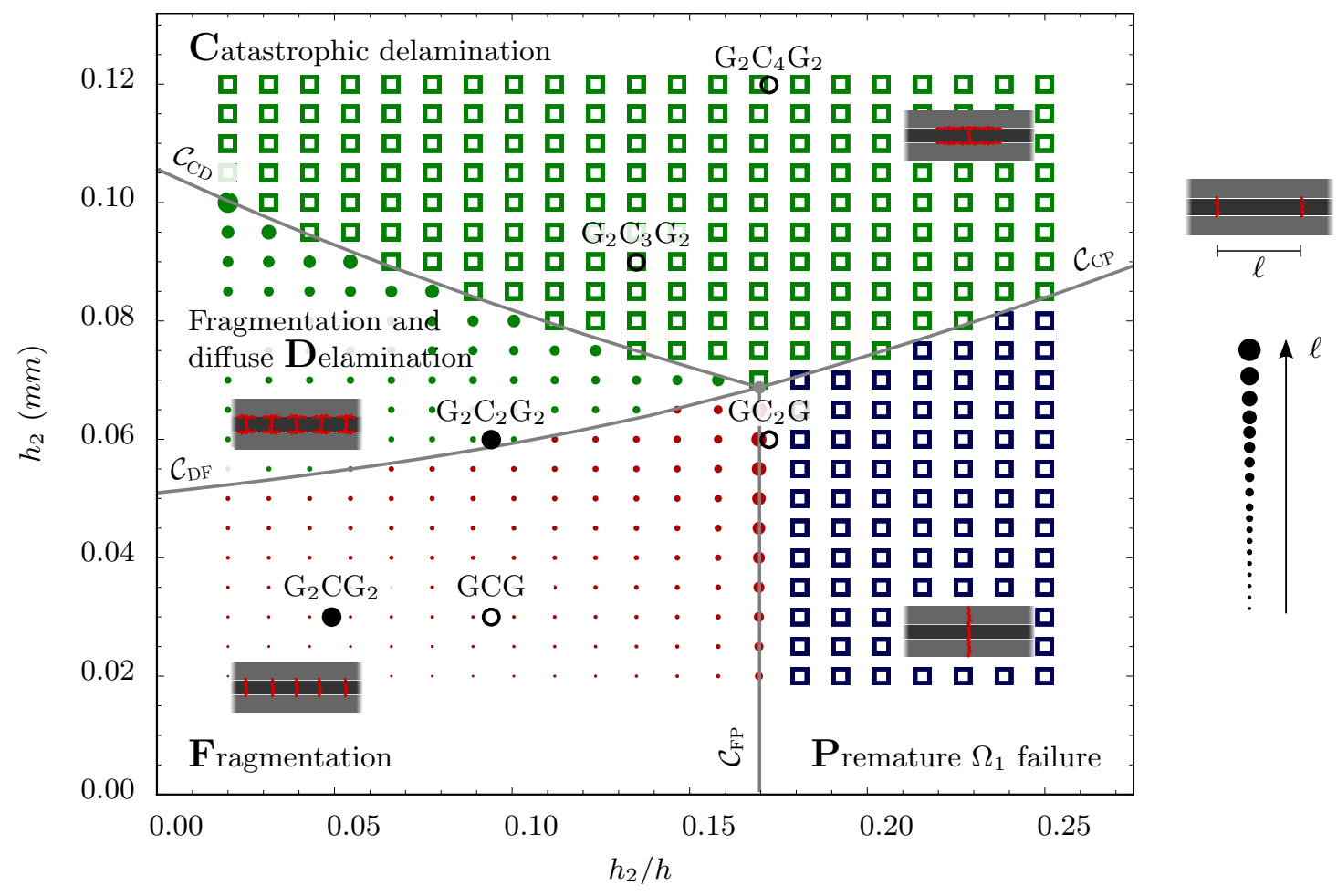

Fig. 10: Damage mode map built with the constitutive parameters of Tab. 2a and Tab. 2b with corresponding limit curves (gray lines) and validated against experiments in [8] of standard glass/carbon laminates (black circles and black dots, this last considered in detail in Sec. 5). Green dots, red dots, green squares and blue squares stand for regions $\mathbf{D}, \mathbf{F}, \mathbf{C}$ and $\mathbf{P}$ respectively. The size of the dots is proportional to the crack spacing length at failure

The material and geometrical properties of these considered laminates are listed in Tab. 2 together with $\bar{\delta}$ and $\delta_{y}=2\left(\bar{\delta}-G_{\text {IIc }} / \bar{\tau}\right)$. The value of $\bar{\delta}$ has been derived by fitting the proposed model against the experimental data in terms of the characteristic crack spacing. The expected failure mechanisms according to the damage mode map in Fig. 10 are: fragmentation for the $\mathrm{G}_{2} \mathrm{CG}_{2}$ layup (Layup 1) and fragmentation with diffuse delamination of the interface for the $\mathrm{G}_{2} \mathrm{C}_{2} \mathrm{G}_{2}$ laminate (Layup 2); this latter confers to the laminate a pseudoductile behavior.

The global structural responses of the laminates, subjected to an uniaxial tension test, are determined by fitting the model in Sec. 4 and compared to the experimental results in Fig. 12. The good agreement between the simple proposed model and the experiments is clear from the figure where the slight differences are likely due to the presence of defects in the real materials, not accounted by the model, and the neglecting of $2 \mathrm{D}$ inplane effects which may play a significant role when strong inhomogeneities and stress localizations occur. It is worth remarking that the abrupt release of energy triggered by the crack initiation may produce a local dynamic process which could end up in overload-

\begin{tabular}{cr}
\hline $\mathrm{E}_{1}$ & $38.7 \mathrm{GPa}$ \\
$\mathrm{E}_{2}$ & $101.7 \mathrm{GPa}$ \\
$\bar{\sigma}_{1}$ & $1548.0 \mathrm{MPa}$ \\
$\bar{\sigma}_{2}$ & $1962.0 \mathrm{MPa}$ \\
\hline
\end{tabular}

(a) Layers mechanical properties

\begin{tabular}{cc}
\hline$\delta_{y}$ & $0.021 \mathrm{~mm}$ \\
$\bar{\delta}$ & $0.025 \mathrm{~mm}$ \\
$\bar{\tau}$ & $67.0 \mathrm{MPa}$ \\
$G_{\text {IIc }}$ & $1.0 \mathrm{~N} / \mathrm{mm}$ \\
\hline
\end{tabular}

(b) Interface mechanical properties

\begin{tabular}{lll}
\hline & Layup 1 & Layup 2 \\
\hline$h_{1}$ & $0.29 \mathrm{~mm}$ & $0.29 \mathrm{~mm}$ \\
$h_{2}$ & $0.03 \mathrm{~mm}$ & $0.06 \mathrm{~mm}$ \\
\hline
\end{tabular}

(c) Thicknesses for the two specimens

Table 2: Mechanical and geometrical properties for the experimental comparisons. The data with gray background are taken from 8

ing the interface and possibly trigger a delamination. This indeed seems to occur in Layup 1 where a diffuse delamination at failure can be seen from Fig. 11a with the carbon layer debonded from the glass layer in the 


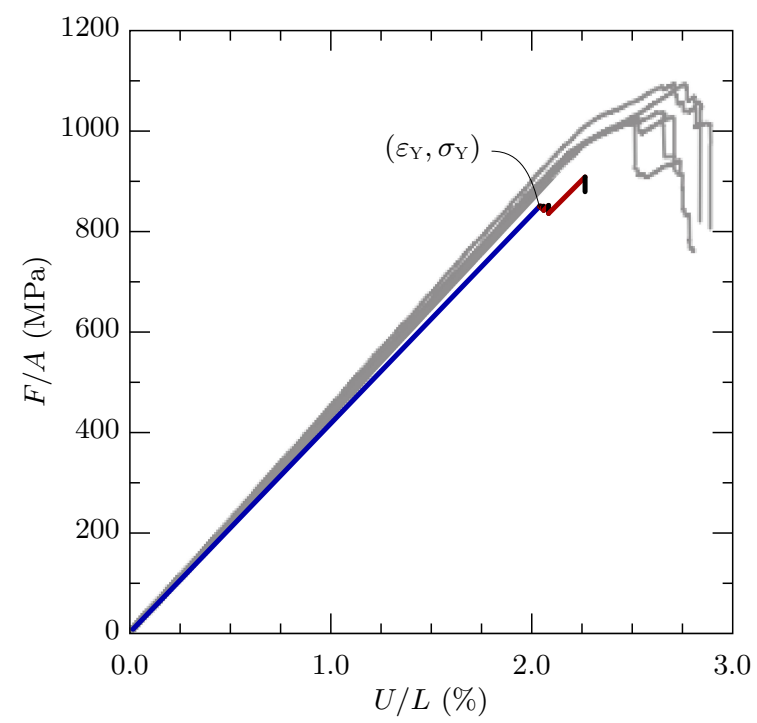

(a) Layup 1

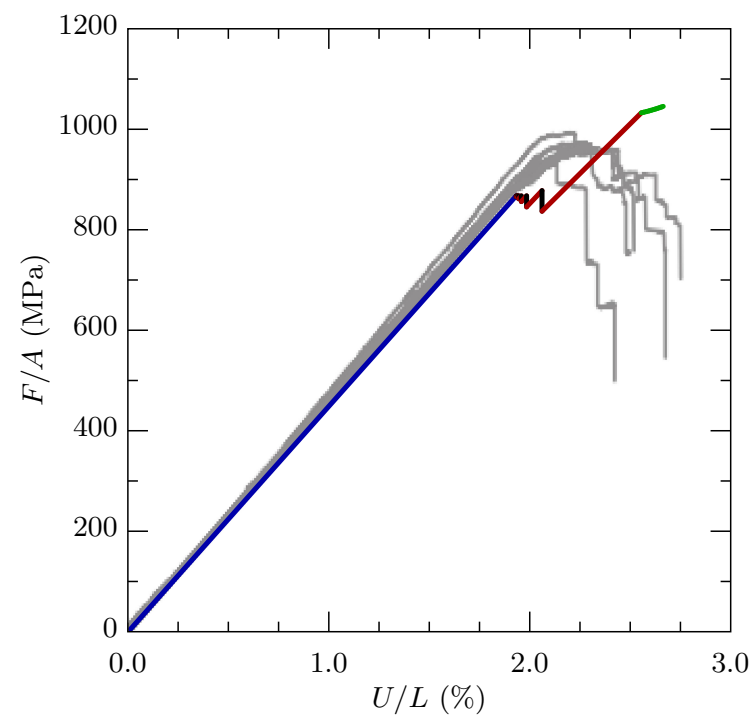

(b) Layup 2

Fig. 12: Comparison of the global responses for a uniaxial traction test between the analytic model and the experiments in [8, Figures 9-10]

surrounding of the cracks, although the prediction by the DMM of a pure fragmentated response.

Despite having small differences in the stress-strain curves, the proposed model is able to predict the crack spacing seen in the experiments whereas the model in 14] with a rigid perfectly plastic interface underestimates it by $50 \%$ (see Tab. 3 and Fig. 11). This is due to the additional parameter introduced in the interface constitutive law with respect to the rigid perfectly plastic model in 14. The values of the constitutive parameters obtained by the fitting and reported in Tab. 2 corresponds to $G_{\mathrm{II} c}^{e}=0.7 \mathrm{~N} / \mathrm{mm}$ and $G_{\mathrm{II} c}^{p}=0.3 \mathrm{~N} / \mathrm{mm}$

\begin{tabular}{llcc}
\hline & $\begin{array}{c}\text { experimental } \\
\text { length }\end{array}$ & $\begin{array}{c}\text { perfectly- } \\
\text { plastic } \\
\text { interface }\end{array}$ & $\begin{array}{c}\text { elasto-plastic } \\
\text { interface }\end{array}$ \\
\hline Layup 1 & $\mathbf{1 . 3} \mathrm{mm}$ & $0.5 \mathrm{~mm}$ & $\mathbf{1 . 3} \mathrm{mm}$ \\
Layup 2 & $\mathbf{2 . 3} \mathrm{mm}$ & $1.0 \mathrm{~mm}$ & $\mathbf{2 . 3} \mathrm{mm}$ \\
\hline
\end{tabular}

Table 3: Crack spacing length comparisons

whereas the corresponding values in 14 are $G_{\mathrm{II} c}^{e}=0.0 \mathrm{~N} / \mathrm{mm}$ and $G_{\text {IIc }}^{p}=1.0 \mathrm{~N} / \mathrm{mm}$. Such a feature of the model would allow a more accurate prediction of the effective bond length as discussed in [10].

\section{Conclusions and Perspectives}

In this work, a one-dimensional analytical model for the description of UD hybrid laminate has been proposed and used to describe the mechanical response and the failure mechanisms of the composite subjected to a tensile loading. The model assumes elastic-brittle layers and cohesive elasto-plastic-brittle interface.

The global response of the composite has been obtained by first deriving the response of a unit-cell and then by iteratively reconstructing the response of the entire laminate. The same algorithm is used to investigate all the possible failure scenarios in terms of the layup geometric properties.

The boundaries of the different damage regions have been fully characterized and closed form expressions for of the limiting curves were derived. As such, the model can provide an efficient and effective way to investigate experimentally new composite layups.

Compared to a model with a rigid-perfectly plastic interface, such as 14, the proposed approach with a cohesive interface has allowed both a more accurate prediction of the crack spacings (underestimated by $50 \%$ in the perfectly plastic model) and a good agreement with the stress-strain curves. These latter evidenced the presence of a pseudo-ductile response. Moreover, the capability of the interface to store elastic energy removes the indeterminacy of the crack location in the low strain layer usually seen in rigid-perfectly plastic models. Indeed when the cracks spacing is large, the location of the next crack is determined in the actual material by imperfections as the stress in the layers is almost constant away from the existing crack. Nevertheless, it is reasonably expected that the introduction of a statistical variation of the parameters affects weakly the final crack spacing.

The proposed analytical approach could be extended by taking advantage of the variational framework used in 3, 4, 1, 2 to two dimensional problems. Crack pat- 
terns similar to the ones observed in [17] with the simultaneous occurrence of debonding and fragmentation are expected.

Acknowledgements The authors acknowledge the support of the Italian National Group of Mathematical Physics (GNFM-

INdAM) through the founding project "Progetto Giovani 2015". 


\section{A Failure-mode function}

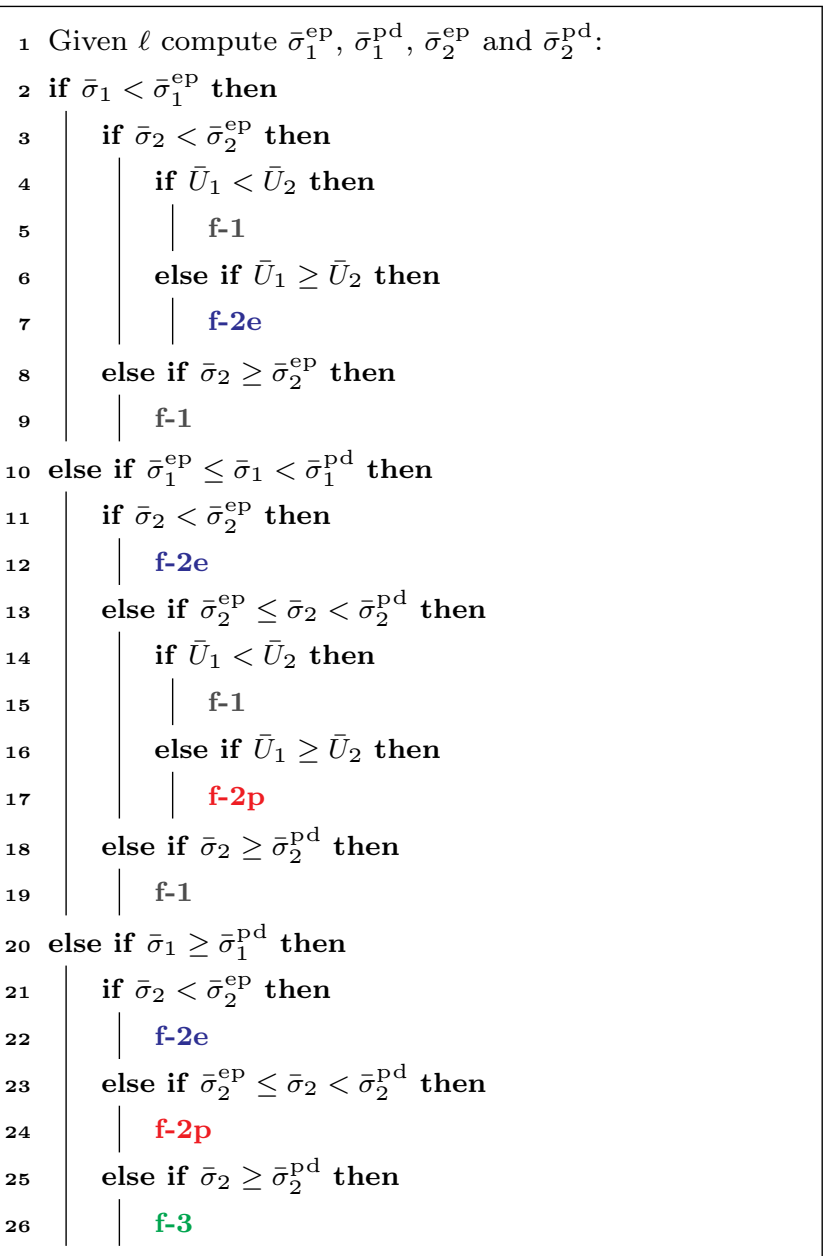

Fig. 13: The failure-mode function (FMF) used in the failure-mode prediction algorithm of Fig. 9. The displacements $\bar{U}_{1}$ and $\bar{U}_{2}$ correspond respectively to the high-strain and low-strain failure displacement of the UC. The labels of the possible failure modes are listed in Tab. 1 


\section{B Equations of Sec. 3.2}

Eq. 27) gives the implicit relation between the applied displacement and the length of the elastically reacting interface during the elasto-plastic stage of the UC. Instead, 28) allows to determine the maximum length $\bar{\ell}_{\mathrm{e}}$ of the elastically reacting interface at which delamination is triggered. Eqs. 29) and (30) give respectively the maximal stresses in the low-strain and high-strain layer as soon as delamination is triggered.

$$
\begin{aligned}
& U_{\mathrm{ep}}\left(\ell_{\mathrm{e}}\right)=\frac{\left(2+\left(1-\ell_{\mathrm{e}}\right)^{2} \alpha+\left(1-\ell_{\mathrm{e}}^{2}\right) \beta\right) \alpha-2 \beta+2 \sqrt{\alpha}\left(\left(1-\ell_{\mathrm{e}}\right) \alpha+\ell_{\mathrm{e}} \beta\right) \operatorname{coth}\left(\ell_{\mathrm{e}} \sqrt{\alpha}\right)}{2 \alpha(\alpha-\beta)} \bar{\tau} \\
& \frac{\alpha\left(\left(\ell_{\mathrm{e}}-1\right) \sqrt{\alpha}\left(2 U(\alpha-\beta)+\left(\ell_{\mathrm{e}}-1\right) \beta \bar{\tau}\right) \cosh \left(\ell_{\mathrm{e}} \sqrt{\alpha}\right)-2\left(U(\alpha-\beta)+\left(\ell_{\mathrm{e}}-1\right) \beta \bar{\tau}\right) \sinh \ell_{\mathrm{e}} \sqrt{\alpha}\right)}{2(\alpha-\beta)\left(\sqrt{\alpha}\left(\left(\ell_{\mathrm{e}}-1\right) \alpha-\ell_{\mathrm{e}} \beta\right) \cosh \left(\ell_{\mathrm{e}} \sqrt{\alpha}\right)-(\alpha-\beta) \sinh \left(\ell_{\mathrm{e}} \sqrt{\alpha}\right)\right)}=\bar{\delta} . \\
& \begin{array}{c}
\bar{\sigma}_{1}^{\mathrm{pd}}:=\frac{\alpha^{3 / 2}\left(2 \bar{U}+\left(-1+\bar{\ell}_{\mathrm{e}}\right)^{2} \bar{\tau}\right) \cosh \left(\bar{\ell}_{\mathrm{e}} \sqrt{\alpha}\right)+2\left(\bar{\ell}_{\mathrm{e}}-1\right) \alpha \bar{\tau} \sinh \left(\bar{\ell}_{\mathrm{e}} \sqrt{\alpha}\right)}{2 \sqrt{\alpha}\left(\left(1-\bar{\ell}_{\mathrm{e}}\right) \alpha+\bar{\ell}_{\mathrm{e}} \beta\right) \cosh \left(\bar{\ell}_{\mathrm{e}} \sqrt{\alpha}\right)+2(\alpha-\beta) \sinh \left(\bar{\ell}_{\mathrm{e}} \sqrt{\alpha}\right)} \\
\bar{\sigma}_{2}^{\mathrm{pd}}:=\frac{(\alpha-\beta)\left(\sqrt{\alpha}\left(2 \bar{U}+\left(1-\bar{\ell}_{\mathrm{e}}\right)^{2} \bar{\tau}\right) \cosh \left(\bar{\ell}_{\mathrm{e}} \sqrt{\alpha}\right)+2\left(1-\bar{\ell}_{\mathrm{e}}\right) \bar{\tau} \sinh \left(\bar{\ell}_{\mathrm{e}} \sqrt{\alpha}\right)\right)}{-\left(2\left(\sqrt{\alpha}\left(\left(1-\bar{\ell}_{\mathrm{e}}\right) \alpha+\bar{\ell}_{\mathrm{e}} \beta\right) \cosh \left(\bar{\ell}_{\mathrm{e}} \sqrt{\alpha}\right)+(\alpha-\beta) \sinh \left(\bar{\ell}_{\mathrm{e}} \sqrt{\alpha}\right)\right)\right)} \\
\quad+\frac{(\alpha-\beta)\left(2\left(\sqrt{\alpha}\left(\left(1-\bar{\ell}_{\mathrm{e}}\right) \alpha+\bar{\ell}_{\mathrm{e}} \beta\right) \cosh \left(\bar{\ell}_{\mathrm{e}} \sqrt{\alpha}\right)+(\alpha-\beta) \sinh \left(\bar{\ell}_{\mathrm{e}} \sqrt{\alpha}\right)\right)\right)}{\left.\left.-\left(1-\bar{\ell}_{\mathrm{e}}^{2}\right) \beta\right) \bar{\tau}\right)}
\end{array}
\end{aligned}
$$

\section{References}

1. Alessi R, Bernardini D (2015) Analysis of localization phenomena in Shape Memory Alloys bars by a variational approach. International Journal of Solids and Structures 73-74:113-133

2. Alessi R, Pham K (2016) Variational formulation and stability analysis of a three dimensional superelastic model for shape memory alloys. Journal of the Mechanics and Physics of Solids 87:150-176

3. Alessi R, Marigo JJ, Vidoli S (2014) Gradient Damage Models Coupled with Plasticity and Nucleation of Cohesive Cracks. Archive for Rational Mechanics and Analysis 214(2):575-615

4. Alessi R, Marigo JJ, Vidoli S (2015) Gradient damage models coupled with plasticity: Variational formulation and main properties. Mechanics of Materials 80, Part $\mathrm{B}(0): 351-367$

5. Ascione L, Feo L, Fraternali F (2005) Load carrying capacity of $2 \mathrm{D} \mathrm{FRP/strengthened} \mathrm{masonry} \mathrm{structures.}$ Composites Part B: Engineering 36(8):619-626

6. Callens MG, Gorbatikh L, Verpoest I (2014) Ductile steel fibre composites with brittle and ductile matrices 61:235244

7. Carrara P, Lorenzis LD (2015) A coupled damageplasticity model for the cyclic behavior of shear-loaded interfaces. Journal of the Mechanics and Physics of Solids 85:33-53

8. Czél G, Wisnom MR (2013) Demonstration of pseudoductility in high performance glass/epoxy composites by hybridisation with thin-ply carbon prepreg. Composites Part A: Applied Science and Manufacturing 52:23-30
9. Esmaeeli E, Barros J (2015) Flexural strengthening of RC beams using Hybrid Composite Plate (HCP): Experimental and analytical study. Composites Part B: Engineering 79:604-620

10. Franco A, Royer-Carfagni G (2014) Effective bond length of FRP stiffeners. International Journal of Non-Linear Mechanics 60:46-57

11. Fukunaga H, Chou T, Fukuda H (1984) Strength of Intermingled Hybrid Composites. Journal of Reinforced Plastics and Composites 3(2):145-160

12. Grace NF, Ragheb WF, Abdel-Sayed G (2003) Flexural and shear strengthening of concrete beams using new triaxially braided ductile fabric. ACI Structural Journal

13. Jalalvand M, Czél G, Wisnom MR (2014) Numerical modelling of the damage modes in UD thin carbon/glass hybrid laminates. Composites Science and Technology 94:39-47

14. Jalalvand M, Czél G, Wisnom MR (2015) Damage analysis of pseudo-ductile thin-ply UD hybrid composites A new analytical method. Composites Part A: Applied Science and Manufacturing 69:83-93

15. Jalalvand M, Czél G, Wisnom MR (2015) Parametric study of failure mechanisms and optimal configurations of pseudo-ductile thin- ply UD hybrid composites. Composites Part A: Applied Science and Manufacturing

16. León Baldelli AA, Bourdin B, Marigo JJ, Maurini C (2012) Fracture and debonding of a thin film on a stiff substrate: analytical and numerical solutions of a onedimensional variational model. Continuum Mechanics and Thermodynamics 25(2-4):243-268

17. León Baldelli AA, Maurini C, Pham K (2015) A gradient approach for the macroscopic modeling of superelasticity in softening shape memory alloys. International Journal of Solids and Structures 52:45-55 
18. Manders PW, Bader MG (1981) The strength of hybrid glass/carbon fibre composites. Journal of Materials Science 16(8):2233-2245

19. van der Meer FP, Dávila CG (2013) Cohesive modeling of transverse cracking in laminates under in-plane loading with a single layer of elements per ply. International Journal of Solids and Structures 50(20-21):3308-3318

20. Mishra R, Militky J, Gupta N, Pachauri R, Behera B (2015) Modelling and simulation of earthquake resistant $3 \mathrm{D}$ woven textile structural concrete composites. Composites Part B: Engineering 81:91-97

21. de Moura MFSF, Fernandes R, Silva FGA, Dourado N (2015) Mode II fracture characterization of a hybrid cork/carbon-epoxy laminate. Composites Part B: Engineering 76:44-51

22. Nguyen H, Mutsuyoshi H, Zatar W (2015) Hybrid FRPUHPFRC composite girders: Part 1 - Experimental and numerical approach. Composite Structures 125:631-652
23. Seo SY, Feo L, Hui D (2013) Bond strength of near surface-mounted FRP plate for retrofit of concrete structures. Composite Structures 95:719-727

24. Swolfs Y, Meerten Y, Hine P, Ward I, Verpoest I, Gorbatikh L (2015) Introducing ductility in hybrid carbon fibre/self-reinforced composites through control of the damage mechanisms. Composite Structures 131:259-265

25. Wetherhold R, Lee F (2001) Shaped ductile fibers to improve the toughness of epoxy-matrix composites. Composites Science and Technology 61(4):517-530

26. Wu ZS (2004) Structural Strengthening and Integrity with Hybrid FRP Composites. In: Proceedings of the Second International Conference on FRP Composites in Civil Engineering (CICE), pp 905-912

27. Zweben C (1977) Tensile strength of hybrid composites. Journal of Materials Science 12(7):1325-1337 